\title{
Preparation and Characterization of Disulfide Functionalized Multi-Walled Carbon Nanotubes for Biomedical Applications
}

\author{
Ke Long Huang (Corresponding author) \\ Institute of Functional Materials and Chemistry, College of Chemistry and Chemical Engineering \\ Central South University, Changsha, Hunan 410083, China
}

Tel: 86-731-887-9850 E-mail: huangkelong@yahoo.com.cn

Ling $\mathrm{Hu}$

Institute of Functional Materials and Chemistry, College of Chemistry and Chemical Engineering

Central South University, Changsha, Hunan 410083, China

E-mail: huling19821216@163.com

Su Qin Liu

Institute of Functional Materials and Chemistry, College of Chemistry and Chemical Engineering

Central South University, Changsha, Hunan 410083, China

\begin{abstract}
To interact with different biologic systems for biomedical applications, chemical modification of carbon nanotubes is always a key step. Disulfide is sensitive to the reductive intracellular environment, and such stimulus-responsive covalent bonds were used to modify carbon nanotubes. After deprotection of $\mathrm{N}$-tert-butoxycarbonyl (Boc) groups of the $\mathrm{N}$-(tert-butyloxycarbonyl) cystamine modified multi-walled carbon nanotubes (MWNTs), positively charged ammonium $\left(\mathrm{NH}_{3}{ }^{+}\right.$) functionalized MWNTs (MWNTs-S-S-NH${ }_{3}^{+}$) with disulfide linkages were obtained. Their surface functional groups and changes of morphologies were characterized by infrared (IR) spectroscope and transmission electron microscope (TEM), respectively. The weight percentage of the immobilized disulfide was estimated by thermogravimetric analysis (TGA). And their cytotoxicity in vitro on cultured human nasopharyngeal SUNE1 cells was evaluated. The biocompatibility of MWNTs was improved compared to that of MWNTs without functional groups.
\end{abstract}

Keywords: Multi-walled carbon nanotubes, Disulfide, Functionalization, In vitro cytotoxicity tests

\section{Introduction}

Recently, carbon nanobubes (CNTs) have attracted considerable attention in biomedical applications. CNTs can act as carriers to deliver a variety of biological and bioactive components such as proteins, peptides, DNAs/ RNAs, drugs and other biological molecules into cells ( Wong et al., 2005, p.6021; Bianco et al., 2005, p.571; Pantarotto et al., 2004, p.5242; Wu et al., 2005, p.6358). To get a kind of well physiologically acceptable material, CNTs should be functionalized firstly. Amines can be further modified with a wide range of biological molecules and therapeutic agents. For example, $\mathrm{NH}_{3}{ }^{+}$-functionalized CNTs $\left(\mathrm{CNTs}^{-} \mathrm{NH}_{3}{ }^{+}\right)$is such a useful material, which can conjugate biomolecules and deliver them into cells. Georgakilas and Prato (Georgakilas et al., 2002, p.3050; Prato et al., 2008, p.60) have reported that amphotericin B, anticancer agent methotrexate, immunogenic peptides have been carried successfully into cells by $\mathrm{CNTs}-\mathrm{NH}_{3}{ }^{+}$.

Disulfide linkage is stable under extracellular physiological conditions but sensitive to the reductive intracellular environment. Introducing disulfide linkages to CNTs could facilitate releasing the targets from modified CNTs. The concentration of intracellular glutathione is adequate to break the disulfide linkages, while glutathione outside the cells has no such ability due to the relative low concentration (Piest et al., 2008, p.308; Lin et al., 2006, p.130). You (You et al., 2007, p.16161) has decorated MWNTs with small molecules of pyridyl-ended disulfide and conjugated bovine serum albumin (BSA) onto the material through a disulfide-exchange reaction, and the BSA could be smartly released from MWNTs in the presence of glutathione in vitro. 
However, there is little information available of functionalizing MWNTs in literature for the purpose of conjugating biomolecules onto CNTs conveniently as well as releasing these molecules smartly. The aim of the work described herein was to functionalize MWNTs, and then to assess the characteristics of toxicity of MWNTs functionalized with disulfide. By this procedure, we can obtain the disulfide functionalities decorated MWNTs with ammonium terminal to endue MWNTs with more convenience for transporting targets and releasing these molecules. The chemical reactions for functionalization of MWNTs are illustrated in Figure 1.

\section{Experimental}

\subsection{Reagents and Measurements}

All solvents and reagents were purchased from commercially available sources and used without further purification unless otherwise stated. The MWNTs (98\%, 40-60 nm outside diameters, 5-15 $\mu \mathrm{m}$ lengths ) were purchased from Shenzhen Nanotech Port Co., Ltd.

Fourier transform infrared (FT-IR) spectra were recorded on a Nicolet 6700 IR spectroscopy. Transmission electron microscopy (TEM) images were acquired on a JEOL JEM-1230 electron microscopy at an accelerating voltage of $100 \mathrm{kV}$. Thermal gravimetric analysis (TGA) was carried out on a Diamond TG-DTA 6300 instrument in flowing Argon at a heating rate of $10{ }^{\circ} \mathrm{C} / \mathrm{min}$ from room temperature to $600{ }^{\circ} \mathrm{C}$.

\subsection{Preparation of functionalized MWNTs}

The N-(tert-butyloxycarbonyl) cystamine, containing disulfide linkage, was synthesized via a procedure reported by Niu ( Niu et al., 2008, p.11988).

Oxidized MWNTs (MWNTs-COOH) were obtained by refluxing MWNTs and concentrated nitric acid for $28 \mathrm{~h}$. The as-prepared MWNTs-COOH $(100 \mathrm{mg})$ were suspended in $15 \mathrm{~mL}$ of DMF solution by sonicating the mixture for $30 \mathrm{~min}$, then EDC $(4.5 \mathrm{mmol})$ and NHS $(4.5 \mathrm{mmol})$ were added to the above suspension and mixed sufficiently. After stirring the mixture for $24 \mathrm{~h}, \mathrm{~N}$-(tert-butyloxycarbonyl) cystamine $(1.14 \mathrm{~g}, 4.5 \mathrm{mmol})$ was added. Then, the reaction was allowed to stir for another $36 \mathrm{~h}$ under Ar gas protection, following by centrifuging, sonication and repeated washing. The products of $\mathrm{N}$-(tert-butyloxycarbonyl) cystamine functionalized MWNTs (MWNTs-S-S-NHBoc) were obtained by drying under vacuum. The Boc protecting groups of the MWNTs-S-S-NHBoc are cleaved by treatment with trifluoroacetic acid in dichloromethane for $2 \mathrm{~h}$ at the room tempreture under $\mathrm{Ar}$ gas protection. After centrifuging and washing, the disulfide functionalized MWNTs (MWNTs-S-S-NH ${ }_{3}^{+}$) were obtained.

\subsection{Toxicity test in vitro}

We studied the toxicity effects of MWNT-S-S- $\mathrm{NH}_{3}{ }^{+}$on human nasopharyngeal SUNE1 cells by MTT assay which was used to determine cell survival in the past few years. SUNE1 cells were incubated with either MWNT-S-S- $\mathrm{NH}_{3}{ }^{+}$or pristine MWNTs. As a control, cells were exposed to physiological saline. The cells were grown in RPMI- 1640 cell culture media at $37{ }^{\circ} \mathrm{C}$ in $5 \% \mathrm{CO}_{2}$ humidified incubator and treated for $24 \mathrm{~h}, 48 \mathrm{~h}$ or $72 \mathrm{~h}$ with doses of MWNT-S-S-NH ${ }_{3}{ }^{+}$increasing from 6.25 to $200 \mu \mathrm{g} / \mathrm{mL}$. The dose of pristine MWNTs used was $50 \mu \mathrm{g} / \mathrm{mL}$.

\section{Results and discussion}

\subsection{IR spectroscopy}

IR spectra of the oxidized MWNTs and positively charged MWNTs are shown in Figure 2. The presence of the added functional groups onto MWNTs is verified. In the spectrum of MWNTs-S-S- $\mathrm{NH}_{3}{ }^{+}$(Figure 2(b)), the characteristic C-H stretching vibration $\left(2923 \mathrm{~cm}^{-1}\right)$, as well as amide I band $\mathrm{C}=\mathrm{O}$ stretching and amide II band $\mathrm{N}-\mathrm{H}$ bending vibration (1624 and $1540 \mathrm{~cm}^{-1}$, respectively) are abserved. These peaks confirm that disulfide were connected to MWNTs successfully.

\subsection{TEM analysis}

Morphology and nano-structures of pristine MWNTs, MWNTs-S-S- $\mathrm{NH}_{3}{ }^{+}$are obserbed by TEM which are shown in Figure 3, TEM observations give definitive proof of the presence of MWNTs-S-S- $\mathrm{NH}_{3}{ }^{+}$in the solution. The MWNTs cut by concentrated nitric acid appear in bundles. After functionalization, the MWNTs-S-S-NH${ }_{3}^{+}$ are shorter and thinner than pristine MWNTs, with diameters of 20-40 nm and lengths of 400-600 nm.

\subsection{TGA analysis}

Thermogravimetric analysis (TGA) measurements of the MWNTs-S-S- $\mathrm{NH}_{3}{ }^{+}$material supplies the quantitative evaluation of the disulfide grafted onto MWNTs (Figure 4). The weight loss of all samples below $200{ }^{\circ} \mathrm{C}$ is mainly attributed to release of water molecules and organic molecules odsorbed by samples. Oxidized MWNTs 
sample (Figure 4(a)) displays a small quantity of weight loss in the region $200-500{ }^{\circ} \mathrm{C}$. A weight loss, corresponding to $21 \%$ for MWNTs-S-S- $\mathrm{NH}_{3}{ }^{+}$sample is observed in the region $200-500{ }^{\circ} \mathrm{C}$ (Figure 4(b)), which is attributed to phrolysis of the disulfide connected with the carboxyl groups of MWNTs. The thermal analysis indicate the surface of MWNTs are grafted with a large number of disulfide.

\subsection{In vitro toxicity}

Insoluble and pristine CNTs have induced cell death in vitro (Cui et al.,2005, p.73; Bottini et al., 2006, p.121; Kiura et al., 2005, p.359). To assess the biological properties of the functionallized MWNTs-S-S- $\mathrm{NH}_{3}{ }^{+}$, we study their toxicity effects on SUNE1 cells. The dates were taken for 24, 48 and $72 \mathrm{~h}$ time points. As can be seen in Figure 5, the percentages of cell death are all less $25 \%$ when cells were treated with MWNTs-S-S- $\mathrm{NH}_{3}{ }^{+}$, showing no significant loss of cell viability compared to untreated cells. According to the $72 \mathrm{~h}$ time point dates, more than $50 \%$ of the cells died in the presence of pristine MWNTs, whereas the vast majority of cells remained alive upon treatment with MWNTs-S-S- $\mathrm{NH}_{3}{ }^{+}$. However, an expected dose-response relationship is observed. At higher concentrations of MWNTs-S-S- $\mathrm{NH}_{3}{ }^{+}$, regardless of 24,48 or $72 \mathrm{~h}$ points dates, MWNTs-S-S- $\mathrm{NH}_{3}{ }^{+}$have continuous reinforcing effects on the cells. The initial studies indicate functionalization of MWNTs reduces toxicity and improves biocompatibility of cells in vitro. These results agree well with previous observations (Sayes et al., 2006, p.135; Lacerda et al., 2006, p.1460).

\section{Conclusions}

We have prepared positively charged MWNTs by attaching disulfide to the nanotubes followed cleavage of the Boc protecting groups. The method is effective for functionalization. Infrared spectroscope characterization, electron microscope measurements and thermal gravimetric analysis reveal that disulfide linkages are introduced onto the surface of MWNTs via amide bonds. The toxicity studies show MWNTs-S-S- $\mathrm{NH}_{3}{ }^{+}$material has only very limited impact upon cell proliferation and cell viability, indicating that functionalization of nanotubes dramatically improves the toxicity profile of this nanomaterials.

Acknowledgements

The financial support from National Natural Science Foundation of China (No. 50772133) and Doctor's Scientific Research and Innovation Foundation of Central South University of China (No.134376211) are greatly acknowledged.

\section{References}

Bianco, A., Kostarelos, K., \& Prato, M. (2005). Biomedical applications of functionalised carbon nanotubes. Chem.Commun., 571-577.

Bottini, M., Bruckner, S., \& Mustelin, T. (2006). Multi-walled carbon nanotubes induce Tlymphocyte apoptosis. Toxicol. Lett., 160, 121-126.

Cui, D. X., Tian, F. R., \& Gao, H. J. (2005). Effect of single wall carbon nanotubes on human HEK293 cells. Toxicol. Lett., 155, 73-85.

dependence of single-walled carbon nanotubes cytotoxicity in vitro. Toxicol. Lett., 161, 135-142.

Georgakilas, V., Tagmatarchis, N., \& Pantarotto, D. (2002). Amido acid functionalisation of water soluble carbon nanotubes. Chem. Commun., 3050-3051.

Kiura, K., Sato, Y., \& Shibata, K. I. (2005). Activation of human monocytes and mouse splenocytes by single-walled carbon nanotubes. J. Biomed. Nanotechnol., 1, 359-364.

Lacerda, L., Bianco, A., \& Kostarelos, K. (2006). Carbon nanotubes as nanomedicines:from toxicology to pharmacology. Adv. Drug Deliv. Rev., 58, 1460-1470.

Lin, C., Zhong, Z., \& Lok, M. C. (2006). linear poly(amido amine)s with secondary and tertiary amino groups and variable amounts of disulfide linkages:Synthesis and in vitro gene transfer properties. J. Controlled Release, $116,130-137$.

Molecular recognition of theophylline derivatives. Langmuir, 24, 11988-11994.

Niu, J., Liu, Z. H., \& Zhang, X. (2008). Surface-Imprinted nanostructured layer-by-layer film for

Pantarotto, D., Singh, R., \& McCarthy, D. (2004). Functionalized carbon nanotubes for plasmid DNA gene delivery. Angew. Chem. Int. Ed., 43, 5242-5246.

Piest, M., Lin, C., \& Mateos Timoneda, M. A. (2008). Novel poly(amido amine)s with bioreducible disulfide linkages in their diamino-units:Structure effects and in vitro gene transfer properties. J. Controlled Release, 130, 
38-45.

Prato, M., Kostarelos, k., \& Bianco, A. (2008). Functionlized carbon nanobubes in drug design and discovery. Acc. Chem. Res.,41, 60-68.

Sayes, C. M., Liang, F., Hudson, J. L., Mendez, J., \& Colvin, V. L. (2006). Functionalization density

Wong, N., Kam, S., \& Dai, H. J. (2005). Carbon nanotubes as intracellular protein transporters:generality and biological functionality. J. AM. CHEM. SOC. 127, 6021-6026.

Wu, W., Wieckowski, S., \& Bianco, A. (2005). Targeted delivery of amphotericin B to cells by using functionalized carbon nanotubes. Angew. Chem. Int. Ed., 44, 6358-6362.

You, Y. Z., Hong, C. Y., \&Pan, C. Y. (2007). Covalently immobilizing a biological molecule onto a carbon nanotube via a stimuli-sensitive bond. J. Phys. Chem. C, 111, 16161-16166.
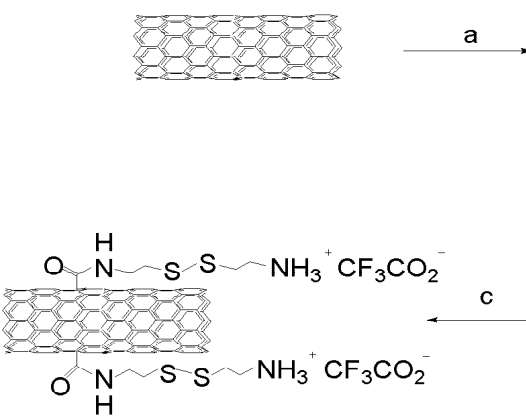

(3)

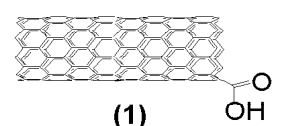

(1)

b

$\mathrm{O} \underset{\mathrm{N}}{\mathrm{H}} \mathrm{S} \sim \mathrm{SHBoc}$

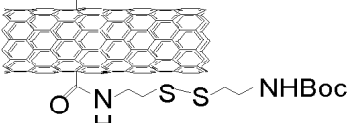

(2)

(a) $\mathrm{HNO}_{3}$, reflux, 28h; (b) $\mathrm{NH}_{2} \mathrm{C}_{2} \mathrm{H}_{4} \mathrm{~S}_{2} \mathrm{C}_{2} \mathrm{H}_{4} \mathrm{NHBoc}$ (4), EDC, NHS in DMF, $25^{\circ} \mathrm{C}, 36 \mathrm{~h}$;

(c) TFA $/ \mathrm{CH}_{2} \mathrm{Cl}_{2}, 25^{\circ} \mathrm{C}, 2 \mathrm{~h}$;

Figure 1. Preparation of disulfide functionalized MWNTs and positively ammonium functionalized MWNTs-S-S-NH ${ }_{3}^{+}$

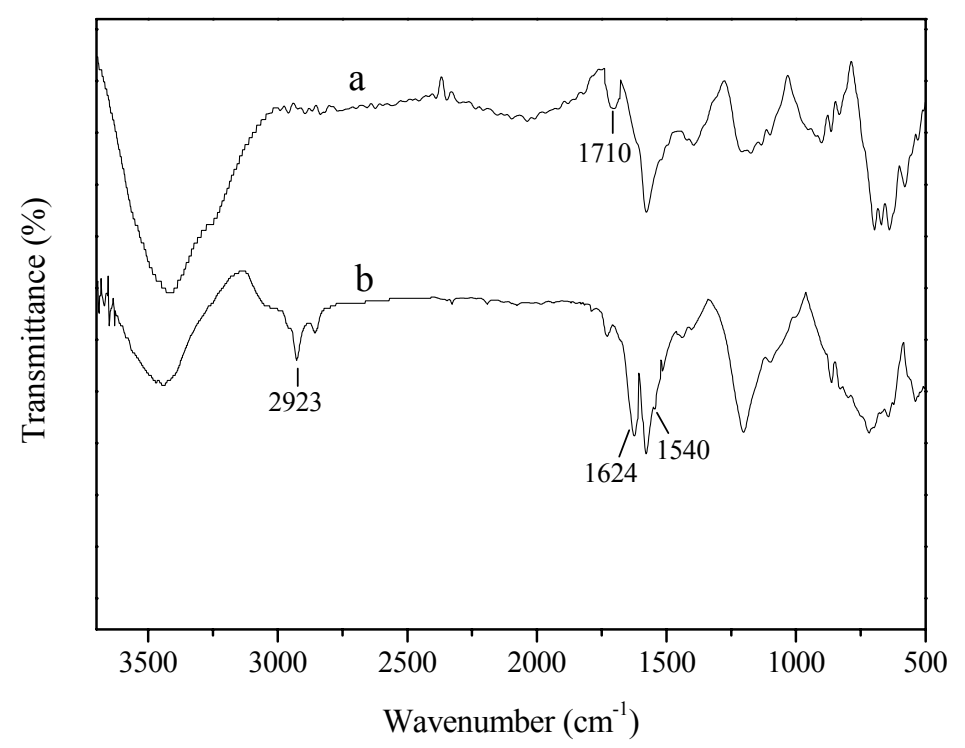

Figure 2. FT-IR spectra of (a) oxidized MWNTs and (b) MWNTs-S-S-NH ${ }_{3}^{+}$ 

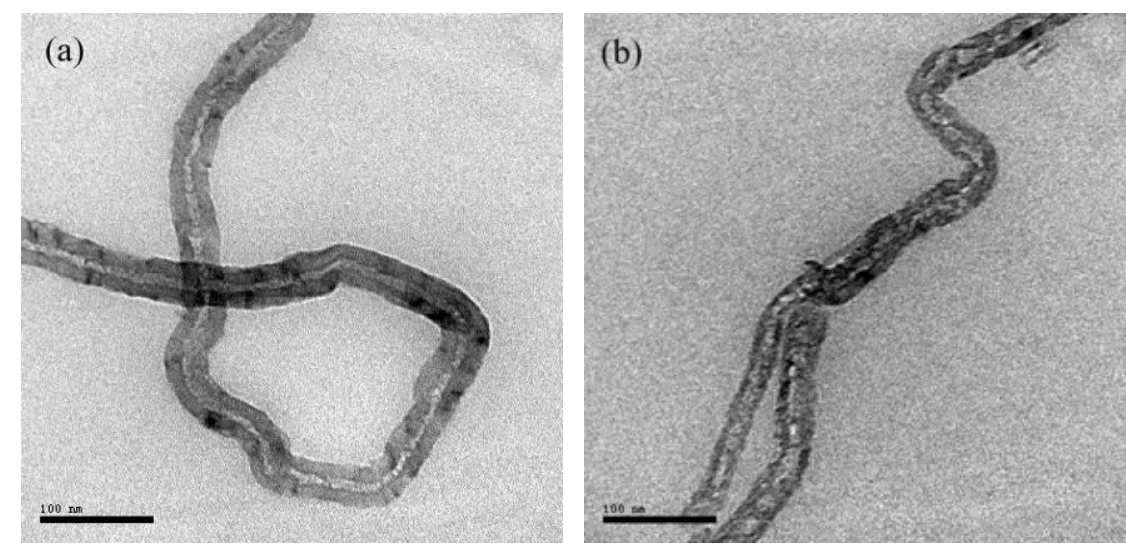

Figure 3. TEM images of (a) pristine MWNTs and (b) MWNTs-S-S- $\mathrm{NH}_{3}{ }^{+}$.The scale toolbars represent $100 \mathrm{~nm}$

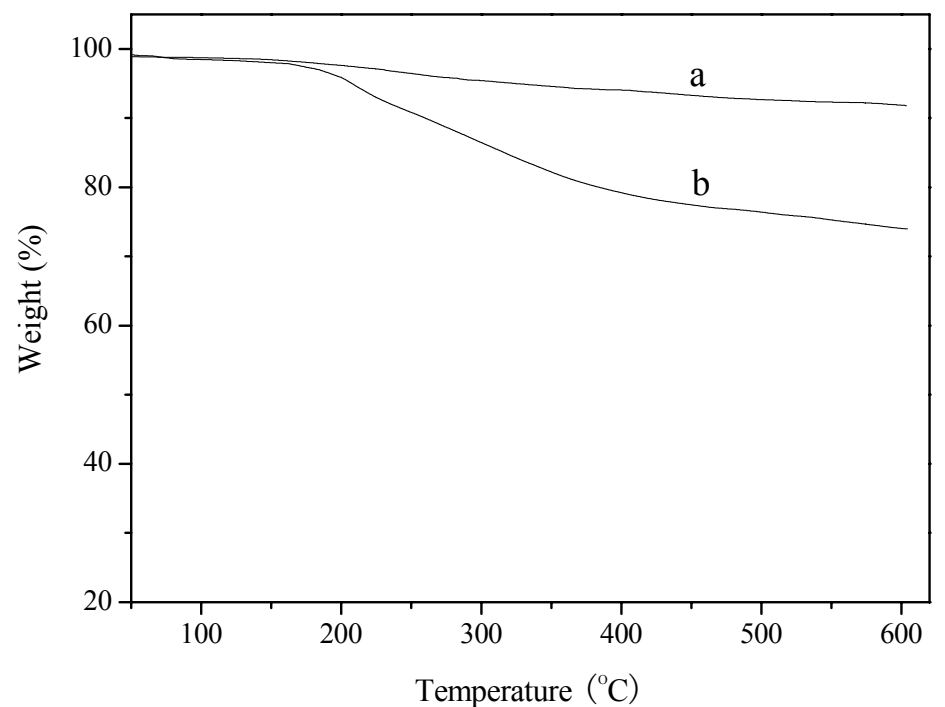

Figure 4. Thermal gravimetric analysis of (a) oxidized MWNTs and (b) MWNT-S-S-NH ${ }_{3}^{+}$

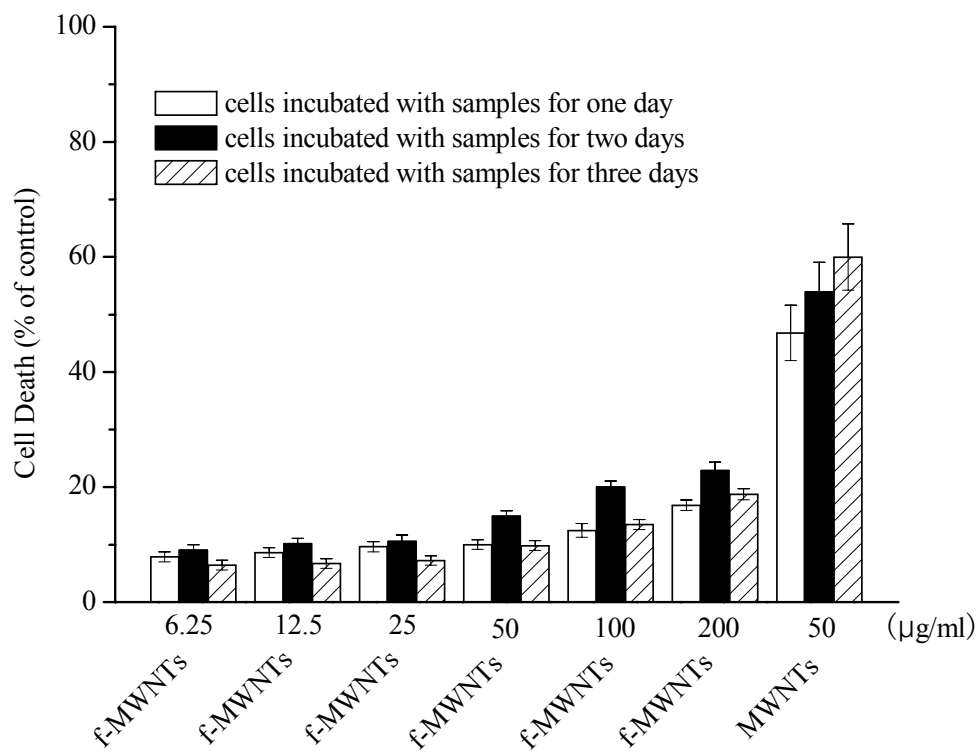

Figure 5. Cell death after treatment with MWNTs-S-S-NH ${ }_{3}^{+}$(f-MWNTs) and pristine MWNTs for $24 \mathrm{~h}, 48 \mathrm{~h}$ or $72 \mathrm{~h}$, respectively 